\section{Super-enhancing discovery}

\section{By Chris Cain, Senior Writer}

Boston researchers have detailed a class of regulatory elements, dubbed super-enhancers, that control the expression of genes, including oncogenes, that are key to determining cellular identity. ${ }^{1,2}$ The findings may explain how some compounds that broadly inhibit particular epigenetic regulators can act selectively on oncogenes and could thus help identify new drug targets in cancer.

The team has founded Syros Pharmaceuticals Inc. to develop compounds that disrupt super-enhancers or the genes they regulate in cancer. $^{3}$

Recent genomewide studies conducted as part of the Encyclopedia of DNA Elements (ENCODE) project have predicted hundreds of thousands to millions of enhancer elements in mammalian genomes that regulate gene expression. ${ }^{4}$ Complementing this effort, whole-genome maps of transcription factor binding sites in individual cell types have found that key regulators of cellular identity tend to work together to form large protein-DNA regulatory complexes at cell type-specific enhancers.

These regulators of cellular identity often control their own expression and that of their co-regulator proteins, forming positive feedback loops that help maintain a particular cellular phenotype.

The best-characterized example comes from embryonic stem cell (ESC) regulators including OCT4, SOX2 and nanog homeobox (NANOG). These sequence-specific DNA-binding factors occupy highly overlapping locations genomewide and control each other's expression and the expression of downstream genes that specify ESC identity. ${ }^{5}$

As expected, all three regulators are required to maintain ESC identity. What was unexpected was the recent demonstration that Mediator, a multisubunit protein complex that is a general coactivator of transcription in all cell types, also was important for determining ESC identity. ${ }^{6}$

Team leader Richard Young thus set out to understand how disrupting Mediator preferentially affects ESC-specific gene expression.

"Mediator is brought to nearly every active gene, yet when we reduce Mediator function in ESCs, it looks exactly like we reduced the levels of the key transcription factors," he said. "How is it that when you perturb a common regulator of gene expression, you can have a very specific kind of biological effect?"

Young is a member of the Whitehead Institute for Biomedical Research, professor of biology at the Massachusetts Institute of Technology and a cofounder of Syros.
To investigate this question, he teamed up with James Bradner, who was investigating a similar conundrum in multiple myeloma (MM) cells. Those cells are particularly sensitive to inhibitors of proteins in the BET bromodomain family such as bromodomain containing 4 (BRD4).

Bromodomain-containing proteins are epigenetic regulators that bind to acetylated lysines on histones; they regulate chromatin remodeling and gene transcription. Previous studies have shown that small molecule BET inhibitors selectively kill some cancer cells, including MM and acute myelogenous leukemia (AML), by downregulating $c-M y c(M Y C)$ expression. ${ }^{7,8}$

"One would think targeting a broadly prevalent chromatin factor would have a general effect on transcription, yet when we treat these cells with a BET inhibitor, we observe that only 50-100 genes are downregulated," Bradner said. "So how is it selective?"

Bradner is an investigator in the Department of Medical
"We went from looking at thousands of genes to looking at the few hundred that have superenhancers. It allows us to focus a tremendous amount of genomic data being produced every day from simplified version that helps us place our bets."

-Richard Young, Whitehead Institute for Biomedical Research ChIP-Seq efforts into a more

Oncology at the Dana-Farber Cancer Institute and assistant professor in the Department of Medicine at Harvard Medical School.

To understand how certain genes might be particularly sensitive to perturbation of general transcriptional regulators, Young's group performed extensive chromatin immunoprecipitation followed by whole-genome sequencing (ChIP-Seq) to map the genomewide binding of numerous proteins. The binding maps included mediator complex subunit 1 (MED1) and BRD4 in MM cells, and MED1, OCT4, SOX2 and NANOG in ESCs.

In ESCs, the researchers identified 8,794 genomic regions that were co-occupied by OCT4, SOX2 and NANOG. What stood out were 231 highly enriched DNA regions within this set that spanned large distances up to $50 \mathrm{~kb}$ in length. These 231 regions had a median length of more than $8.5 \mathrm{~kb}$, whereas the other 8,563 regions had a median length of $0.7 \mathrm{~kb}$, which is what would be expected for a typical enhancer.

Notably, these long regions were found near genes known to be critical regulators of cell identity, including OCT4, SOX2 and NANOG.

Young dubbed these regions super-enhancers because of their length and ability to drive transcription to higher levels than typical enhancers. In addition, super-enhancer activity was more sensitive to knockdown of MED1 than typical enhancers, and super-enhancers bound at least 10 -fold the amount of MED1 as typical enhancers. Super-enhancers were also quite heterogeneous, spanning a wide range of lengths and displaying distinct patterns of transcription factor binding.

The researchers saw similar results in an MM cell line driven by a translocation upstream of $c-M y c$. About 8,000 enhancer regions were bound by BRD4 and MED1, and of those, 308 super-enhancers were identified that were on average 15 -fold larger than traditional 
enhancers and that bound 18-fold more MED1 and 16-fold more BRD4.

Many genes associated with MM pathology were regulated by superenhancers, including $c-M y c$.

When treated with JQ1, a BRD4 inhibitor, super-enhancers were, on average, more depleted of BRD4 than typical enhancers, and genes controlled by super-enhancers exhibited, on average, moderate but statistically lower expression than genes regulated by typical enhancers. The expression of certain genes associated with super-enhancers, most notably $c-M y c$, was dramatically reduced by JQ1 treatment.

Young said a possible explanation for the increased sensitivity of super-enhancers is that they are regulated by more cooperative binding interactions than typical enhancers due to their size, which would render them more sensitive to changes in transcription factor concentrations.

To confirm the widespread existence of super-enhancers, the researchers generated and analyzed ChIP-Seq data from multiple additional cell lines. In glioblastoma multiforme (GBM) and small-cell lung cancer (SCLC) samples, ChIP-Seq identified large super-enhancer regions near multiple tumor-associated genes.

In an analysis of existing ChIP-Seq data from a series of normal cells of varying types, super-enhancers were found upstream of key genes associated with the identity of each respective cell type.

Results were published in two separate Cell papers. One focused on ESCs and normal cells, ${ }^{1}$ and the other focused on cancer cells. ${ }^{2}$ Young was corresponding author of both studies, and Bradner was a coauthor on the cancer study.

"The papers are strong on concept and data. People may have seen this

type of binding before, but the authors took away a new concept from this data, and concepts are important in science," said Kristian Helin, director of the Biotech Research \& Innovation Centre at the University of Copenhagen and CSO of EpiTherapeutics ApS.

\section{Target hunting}

Young and Bradner founded Syros last year to apply insights gained from mapping super-enhancers to cancer drug development. Earlier this month, the company exited stealth mode with a $\$ 30$ million series $\mathrm{A}$ round led by ARCH Venture Partners and Flagship Ventures. ${ }^{3}$

CEO Nancy Simonian said the company is taking a two-pronged strategy that involves targeting super-enhancers themselves and identifying new targets that may have been previously missed by expression analysis.

"One of the important discoveries in this work is the demonstration that if you target a component of super-enhancers, you can get selective effects. It allows you to think you can disrupt components of enhancers and get preferential disruption of super-enhancer function," she said.

Simonian said the company is interested in targeting transcriptional regulatory machinery, which could include kinases. Thus, the company's third cofounder, Nathanael Gray, was recruited for his expertise in kinase inhibitor biochemistry. Gray is professor of biological chemistry and molecular pharmacology at Harvard Medical School.

Simonian added that the company is not developing BRD4 inhibitors and emphasized that JQ1 was used solely as proof of concept that super-enhancers could be selectively inhibited. In 2011, Bradner founded Tensha Therapeutics Inc. to develop derivatives of JQ1, which are in preclinical development.

Young suggested that the approach taken to characterize the precise functions of JQ1 could serve as a model for understanding the actions of compounds that inhibit other transcriptional regulators, including epigenetic targets. "Everyone seems very focused on the development of drugs against 'epigenetic' regulators, but for any component of enhancers that can be drugged, whether a histone-modifying enzyme or a kinase, it is now possible to map out the logic of control pathways associated with enhancers," he said.

Helin agreed that this level of understanding is critical for epigenetic drug discovery programs and noted that companies including EpiTherapeutics already are routinely using techniques including ChIP-Seq to generate this information. "Biotechs have to do these types of experiments-it is obviously important for understanding the direct effects of your drug," he said.

He wanted to see more work characterizing both super-enhancers and the effect of BET inhibition in additional MM cell lines, which can display varying levels of sensitivity to BET inhibitors and can drive $c-M y c$ expression through a variety of distinct genetic mechanisms.

Bradner said additional experiments are ongoing to characterize super-enhancers in additional MM cells that show a range of sensitivities to JQ1, including cells with different genomic alterations that drive $c-M y c$ expression.

Young said that beyond targeting the super-enhancers themselves, super-enhancers could be used to hone in on key cell fate regulators or drug targets that may have been missed by other approaches.

"We went from looking at thousands of genes to looking at the few hundred that have super-enhancers," he said. "It allows us to focus a tremendous amount of genomic data being produced every day from ChIP-Seq efforts into a more simplified version that helps us place our bets."

Helin said the real demonstration of the utility of super-enhancer mapping will come when the approach uncovers new functional biology. "The real test is to say, can you turn this idea around, and can you find the transcription factor that is driving cell fate in a system where we don't already know the identity of that factor," he said.

Syros has exclusively licensed undisclosed IP covering biochemical assays and bioinformatics approaches used to identify super-enhancers, in addition to undisclosed IP from Whitehead and Dana-Farber covering two small molecule programs against undisclosed targets.

Cain, C. SciBX 6(16); doi:10.1038/scibx.2013.379

Published online April 25, 2013

\section{REFERENCES}

1. Whyte, W.A. et al. Cell; published online April 11, 2013; doi:10.1016/j.cell.2013.03.035

Contact: Richard A. Young, Whitehead Institute for Biomedical Research, Cambridge, Mass.

e-mail: young@wi.mit.edu 
2. Lovén, J. et al. Cell; published online April 11, 2013; doi:10.1016/j.cell.2013.03.036

Contact: Richard A. Young, Whitehead Institute for Biomedical Research, Cambridge, Mass. e-mail: young@wi.mit.edu

3. Cain, C. BioCentury 21(15), A12; April 15, 2013

4. Anonymous. Enhancer discovery and characterization. Nature ENCODE (accessed April 24, 2013)

5. Young, R.A. Cell 144, 940-954 (2011)

6. Kagey, M.H. et al. Nature $467,430-435$ (2010)

7. Delmore, J.E. et al. Cell 146, 904-917 (2011)

8. Zuber, J. et al. Nature 478, 524-528 (2011)
COMPANIES AND INSTITUTIONS MENTIONED ARCH Venture Partners, Chicago, III. Dana-Farber Cancer Institute, Boston, Mass. EpiTherapeutics ApS, Copenhagen, Denmark Flagship Ventures, Cambridge, Mass.

Harvard Medical School, Boston, Mass.

Massachusetts Institute of Technology, Cambridge, Mass. Syros Pharmaceuticals Inc., Watertown, Mass. Tensha Therapeutics Inc., Cambridge, Mass. University of Copenhagen, Copenhagen, Denmark Whitehead Institute for Biomedical Research, Cambridge, Mass. 